\title{
Non-linear radial pulsation models for the early-type helium stars V652 Her and BX Cir
}

\author{
P. Montañés Rodríguez and C. S. Jeffery
}

\author{
Armagh Observatory, College Hill, Armagh BT61 9DG, Northern Ireland \\ Received 18 September 2001 / Accepted 2 January 2002
}

\begin{abstract}
We report new non-linear pulsation models of the helium stars V652 Her and BX Cir. Linear theory has previously shown their pulsations to be due to iron-group bump instability. Recent high-resolution spectroscopic observations have provided high-precision measurements of their radial velocity curves and of their radii. Their masses remain less well determined. A hydrodynamic code including recent OPAL opacity data has been used to construct the models. These are compared with the observational data. In particular, we attempt to reproduce accurately the observed radial velocity and luminosity curves. The results impose additional constraints on those stellar dimensions, including mass, which remain poorly determined by observation. Final results show a model for V652 Her which reproduce the observed velocity and luminosity curves with $0.7 M_{\odot}$ and $23400 \mathrm{~K}$. For BX Cir, the mass must lie between 0.50 and $0.38 M_{\odot}$ if the temperature is in the range $22400-24000 \mathrm{~K}$. However, the luminosity of the models is smaller than that measured directly by a factor of two.
\end{abstract}

Key words. stars: helium - stars: individual: V652 Her - stars: individual: BX Cir - stars: oscillations stars: early-type

\section{Introduction}

Extreme helium early-type stars are located in a region on the H-R diagram between 10000 and $25000 \mathrm{~K}$ and with $\log L / L_{\odot} \gtrsim 3$. Although this locus coincides with that of massive main sequence stars, they are low mass stars $\left(\lesssim 1.0 M_{\odot}\right)$ in an advanced stage of evolution. Their unusually low surface hydrogen abundance, less than $1 \%$ (Drilling et al. 1998; Jeffery et al. 2001) is a question not entirely solved by stellar evolution theory.

Several theories have been proposed to explain their origin. The most recent hypothesis, explain the evolution of a merged binary helium white dwarf to become a low-mass helium star (Saio \& Jeffery 2000). But evolution models strongly depend on the stellar parameters, in particular, the stellar mass and metallicity have to be accurately known.

A few low-mass (early-type) helium stars show periodic light variations due to stellar pulsations (Kilkenny \& Koen 1995; Lynas-Gray et al. 1984; Jeffery \& Saio 1999; Kilkenny et al. 1999) which provide a direct way to measure their mass, metallicity and position within the H-R diagram. At present just two of these, V652 Her and BX Cir (BD + 13 3224 and LSS 3184) have been identified as purely radial pulsators.

Send offprint requests to: P. Montañés Rodríguez, e-mail: pmr@star.arm.ac.uk
The instability strip for extreme helium stars was first discovered, using OPAL opacities and linear analysis, by Saio (1995). He found that pulsation models become unstable due to the iron-group ionization opacity peak at around $2 \times 10^{5} \mathrm{~K}$ (iron-group bump mechanism). For early-type helium stars the instability boundaries were approximately located between $T_{\text {eff }}=25000$ and $16000 \mathrm{~K}$ and between $L=300$ and $1000 L_{\odot}$ with small variations depending on stellar mass and metallicity.

Non-linear analyses of V652 Her have been previously carried out by Fadeyev \& Lynas Gray (1996), providing radial velocity and luminosity curves for $0.72 M_{\odot}$ models in good agreement with observational data. They also studied the variations of velocity and luminosity amplitudes within the instability strip, finding that the boundaries are close to those proposed by Saio (1995).

Recent observations of V652 Her (Jeffery et al. 2001) using higher resolution have provided a lower value for its mass. Also a more accurate measurement of its abundances gives a higher nitrogen abundance than previous results, as well as a carbon abundance smaller than solar. New observations have also provided better estimates of the mass and effective temperature of BX Cir (Woolf \& Jeffery 2000).

The goal of this paper is to constrain the stellar parameters and in particular, the mass, effective temperature and metal abundances of V652 Her and BX Cir, by comparing new non-linear models with the recent 
Table 1. Observational parameters for V652 Her and BX Cir. For V652 Her, right column shows the dimensions provided by recent observations, where two different methods were applied to determine the effective temperature (see text). Left column shows the dimensions determined by previous observations. Luminosity is calculated from the observed radius and temperature.

\begin{tabular}{|c|c|c|c|}
\hline & $\begin{array}{ll} & \text { V65 } \\
\text { previous } & \end{array}$ & $\begin{array}{l}52 \text { Her } \\
\text { recent }\end{array}$ & $\begin{array}{l}\mathrm{BX} \text { Cir } \\
\text { recent }\end{array}$ \\
\hline$\Pi / \mathrm{d}$ & $0.108^{(1)}$ & & $0.1066^{(4)}$ \\
\hline$M / M_{\odot}$ & $0.7_{-0.3}^{+0.4}(2)$ & $\begin{array}{l}a 0.17 \pm 0.05^{(3)} \\
b 0.59 \pm 0.18^{(3)}\end{array}$ & $0.42 \pm 0.12^{(5)}$ \\
\hline$T_{\text {eff }} / \mathrm{kK}$ & $23.45 \pm 0.13^{(2)}$ & $\begin{array}{l}a 22.93 \pm 0.01^{(3)} \\
b 20.95 \pm 0.07^{(3)}\end{array}$ & $23.30 \pm 0.7^{(6)}$ \\
\hline$R / R_{\odot}$ & $1.98 \pm 0.21^{(2)}$ & $\begin{array}{l}a 1.26 \pm 0.00^{(3)} \\
b 2.31 \pm 0.02^{(3)}\end{array}$ & $2.31 \pm 0.10^{(5)}$ \\
\hline $\log L / L_{\odot}$ & $3.03 \pm 0.21$ & $\begin{array}{l}a 2.59 \pm 0.00^{(3)} \\
b 2.96 \pm 0.01^{(3)}\end{array}$ & $3.15 \pm 0.09$ \\
\hline $\log g / \mathrm{cm} \mathrm{s}^{-2}$ & $3.7 \pm 0.2^{(2)}$ & $3.46 \pm 0.05^{(3)}$ & $3.35 \pm 0.1^{(6)}$ \\
\hline
\end{tabular}

observational data, as well as to analyze the variations of the light and velocity curves for this range of masses and effective temperatures.

\section{Observational parameters for $\mathrm{V} 652 \mathrm{Her}$ and BXCir}

Since the discovery of V652 Her as a short period variable by Landolt (1975), several observations have been carried out to determine its stellar properties with better accuracy. Initially, it was identified as a radially pulsating star by Hill et al. (1981). A more precise measurement of its radius was obtained by Lynas-Gray et al. (1984) by including ultraviolet spectroscopy. Subsequent studies of the light curves and spectrum of V652 Her are summarized in Tables 1 and 2, and Fig. 1. In particular, Table 1 shows the recent observed parameters (Jeffery et al. 2001) measured $(a)$ by visual magnitude and $(b)$ by ultraviolet spectrophotometry, although the unrealistically low mass obtained by the first method, causes us to choose $(b)$ parameters, where systematic errors are probably smaller.

A second pulsating helium star, BX Cir, was discovered by Drilling (1980) and, as V652 Her, was predicted to pulsate due to its location in the instability region (Saio 1995). Kilkenny \& Koen (1995) measured a period of 0.1066 days. An atmospheric analysis by Drilling et al. (1998) reported the dimensions and abundances shown in Tables 1 and 2. Subsequent analysis by Woolf \& Jeffery (2000) measured BX Cir's radial velocity, angular radius and stellar mass, providing the results also shown in Table 1.

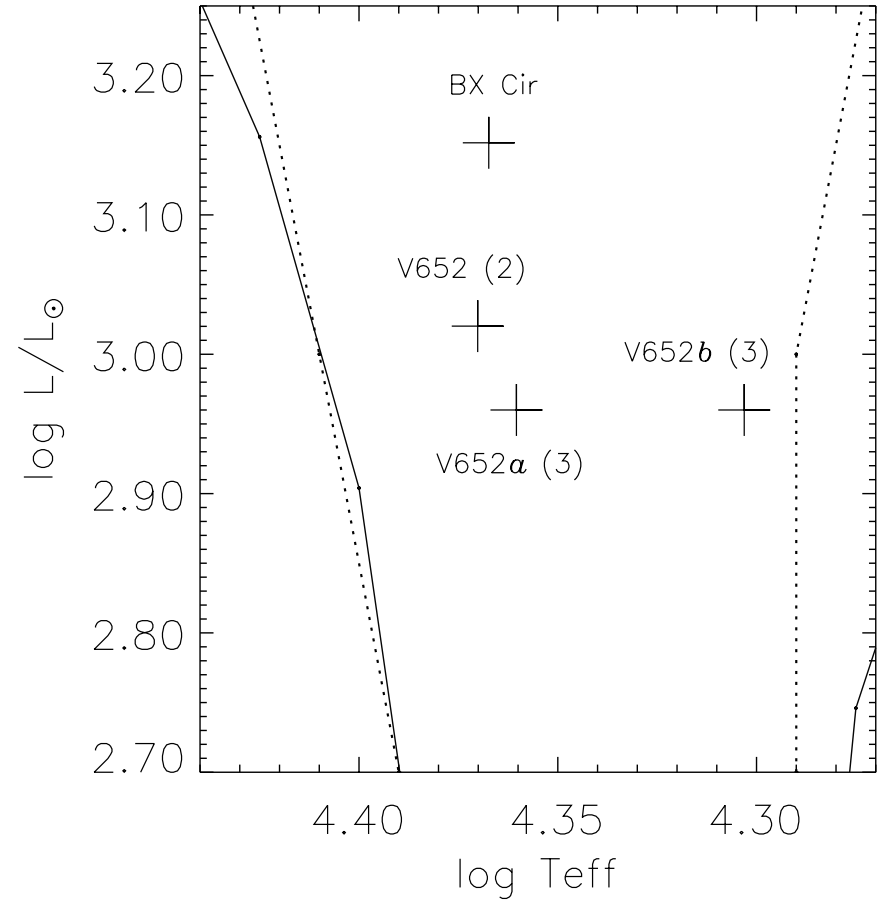

Fig. 1. H-R diagram showing the location of V652 Her and BX Cir according to references given in Table. 1. Stellar luminosity was calculated from the provided effective temperature and radius. The boundaries of the region of pulsational instability due to the iron-group bump mechanism for $0.5 M_{\odot}$ (solid line) and $0.7 M_{\odot}$ (dotted line) for metallicity $Z=0.01$ are also shown.

Table 2. Photometric abundances for V652 Her and BX Cir. Comparisons are also given for the Sun. Note the excess of nitrogen reported for V 652 and the excess of carbon for BX Cir.

\begin{tabular}{cccc}
\hline & Sun & $\mathrm{V} 652 \mathrm{Her}^{(3)}$ & $\mathrm{BX} \mathrm{Cir}^{(6)}$ \\
$n_{\mathrm{H}}$ & $0.90976^{(7)}$ & 0.01159 & 0.00015 \\
$n_{\mathrm{He}}$ & $0.08891^{(7)}$ & 0.98626 & 0.99590 \\
$n_{\mathrm{N}}$ & $0.00010^{(7)}$ & 0.00139 & 0.00052 \\
$n_{\mathrm{C}}$ & $0.00035^{(8)}$ & $5.55 \times 10^{-5}$ & 0.00301 \\
$n_{\mathrm{Fe}}$ & $2.75 \times 10^{-5(9)}$ & $3.12 \times 10^{-5}$ & $9.51 \times 10^{-6}$ \\
$n_{\mathrm{Z}}$ & $0.00133^{(7)}$ & 0.00216 & 0.00394 \\
\hline
\end{tabular}

${ }^{3}$ Jeffery et al. (2001), ${ }^{6}$ Drilling (1998), ${ }^{7}$ Anders \& Grevesse (1989), ${ }^{8}$ Stürenburg \& Holweger (1990), ${ }^{9}$ Holweger et al. (1990).

\section{The method of computation}

A non-linear pulsation code (Bridger 1984) based on the description by Christy (1967) has been used to model radial oscillations. Equations of motion and continuity were solved using the explicit finite-difference scheme, whereas the energy equation was treated implicitly with the CrankNicholson scheme. Each pulsation model represents a fundamental mode radial pulsator and is characterized by four parameters, $L, M, T_{\text {eff }}$ and chemical abundances. 
The initial model of the stellar envelope was calculated in hydrostatic equilibrium. The equations were integrated inward from the surface, using approximately 5000 integration points, after choosing an outer-zone mass and a zone mass ratio $(\approx 1.1)$. The number of mass zones considered in the different hydrodynamic models varies between 70 and 80 .

Convection was ignored and chemical composition was assumed to be constant. The inner boundary of the pulsating envelope was considered to be a rigid sphere radiating with constant luminosity. At the surface, the standing wave or total reflection boundary condition was applied $\left(P_{\text {tot }}=0\right)$. The inner boundary must be at a radius of less than one tenth of the stellar radius and must have a temperature $\sim 1 . \times 10^{7} \mathrm{~K}$.

The static model was perturbed using a radius dependent velocity distribution,

$U_{i, 1}=\mathrm{A}_{1}\left(\frac{R_{i, 1}}{R_{n, 1}}\right)^{5}+6.0\left(\frac{R_{i, 1}}{R_{n, 1}}\right)^{7}\left(\mathrm{~km} \mathrm{~s}^{-1}\right)$.

Here $R_{i, 1}$ is the initial radius in the $i$ th zone, $R_{n, 1}$ is the initial radius at the surface and the amplitude $A_{1}$, varies for different models and have typical values in the range 5-20 $\mathrm{km} \mathrm{s}^{-1}$. This was done to reduce computing time, rather than assume $U_{i, 1}=0$.

An artificial viscosity $Q_{i}$ was introduced as a dissipative pressure following the Richtmyer-Morton method, with the equation described by Stellingwerf (1975).

The adopted viscosity parameters were $C_{\mathrm{Q}}=4.0$, following previous work for helium-rich envelopes (see Saio 1985 ), and $\alpha_{\mathrm{v}}=0.1$. However, a deeper analysis of the propagation of shock waves in such media would be interesting, since a different viscosity can modify the shape, and the amplitude, of the velocity and luminosity curves.

For temperatures lower than $10 \mathrm{kK}$ the radiative opacity was calculated using hydrogen-deficient Alexander's (1994) opacities, with a standard solar mixture of metals (see http://web.physics.twsu.edu/alex/pub/ G93.txt). OPAL opacity tables (Iglesias \& Rogers 1996) were used for temperatures higher than $10 \mathrm{kK}$. These were initially generated in the OPAL web site (http://www-phys.llnl.gov/VDiv/OPAL/) to have solar mixture of metals with enhanced nitrogen or carbon. Final tables were smoothed and interpolated using adapted OPAL software.

The hydrodynamic models are characterized by their maximum kinetic energy per pulsation cycle, E. For a model with $n$ mass zones, the kinetic energy for each temporal step $I$, is given by

$E_{I}=\frac{1}{2} \sum_{i=1}^{n} \Delta M_{i} U_{i}^{2}$

$E$ is the maximum value of $E_{I}$ over a pulsation cycle. As a consequence of the initial perturbation, $E$ decreases initially in some models. In contrast, in the case where $U_{i, 1}=0, E$ increases initially. To ensure that our hydrodynamic calculations had converged, models were continued

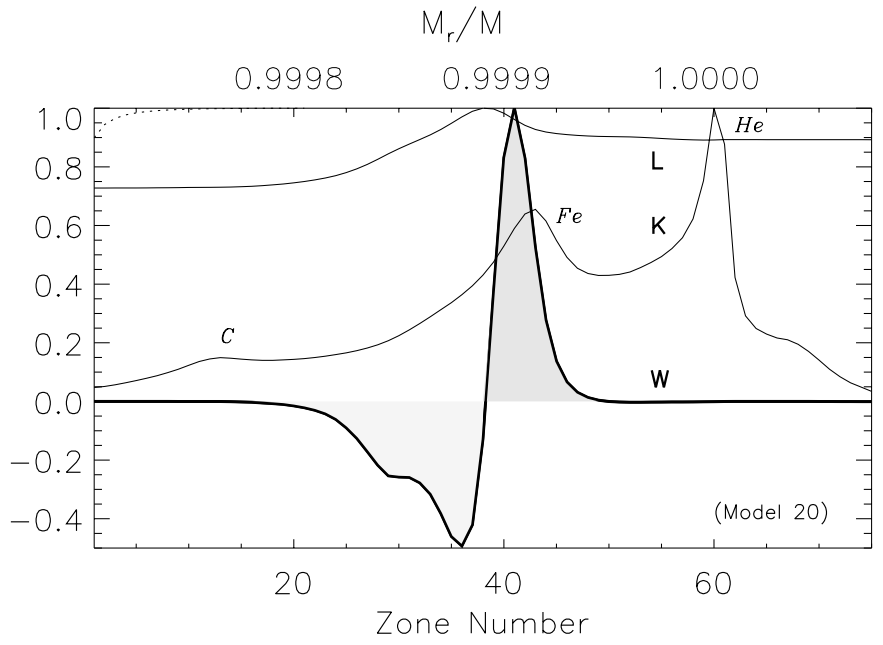

Fig. 2. The distributions of pulsational work integral, divided by its maximum value (thicker line $W$ ), luminosity $(L)$ and opacity $(K)$ at luminosity maximum, for model 20.

until $E$ reached a constant value and therefore the pulsation had reached a limiting amplitude. However, some unstable models which did not reach limiting amplitudes were used to find the pulsation periods and to establish limits to the amplitudes of the pulsation.

In general, the distribution of the pulsational work integral for models located within the boundaries of the instability region, has a maximum value deeper in the envelope than for models located in the Cepheid instability strip. This is due to the effect of the iron-group bump, as is shown in Fig. 2. The driving region, where the pulsation work is positive, coincides with the region of maximum luminosity (around zone 40) and with the iron-group opacity peak.

\section{Models}

Homologous stellar pulsations obey the period-mean density relation

$\Pi=Q \sqrt{\frac{R^{3}}{M}}$.

$\Pi$ is the pulsation period, $R$ and $M$ are the stellar radius and mass and $Q$ is a factor depending on the structure of the stellar envelope.

Since luminosity and effective temperature are related by the identity,

$$
\begin{aligned}
L & =4 \pi \sigma R^{2} T_{\mathrm{eff}}^{4} \\
& =T_{\mathrm{eff}}{ }^{4} 4 \pi(\Pi / Q)^{4 / 3} M^{2 / 3}
\end{aligned}
$$

then,

$\log L=4 \log T_{\text {eff }}+a_{1}$,

where, $a_{1}=\log \left(4 \pi \sigma(\Pi / Q)^{4 / 3} M^{2 / 3}\right)$.

We note that models which have the measured radii and masses of V652 Her and BXCir do not give a period corresponding to that observed. However, period is the 


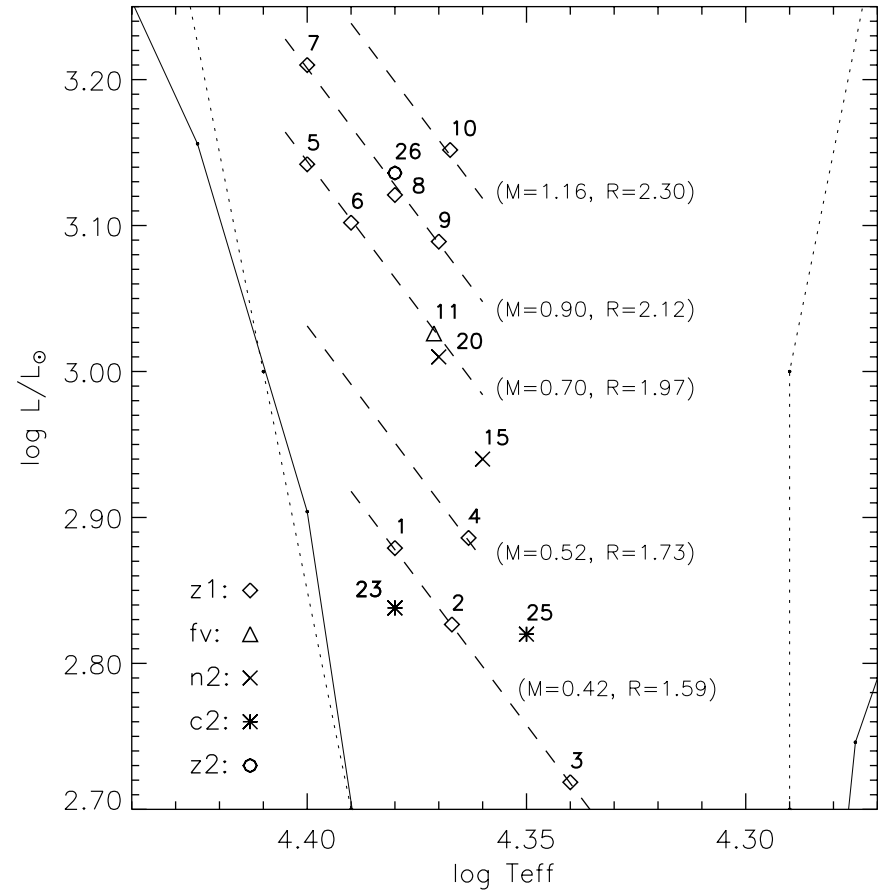

Fig. 3. H-R diagram showing the models calculated. Different symbols show chemical mixtures used in Table 3. Boundaries of the instability region for this range of masses and chemical composition are also shown.

best observed constraint. For this reason, we have chosen to investigate families of models obeying Eq. (6), with $\Pi=0.107 \mathrm{~d}$ to within \pm 0.002 days and masses in the range 0.38-1.16 $M_{\odot}$. We have also investigated models with a range of chemical composition, including a standard mixture of 21 metals (Grevesse \& Noels 1993) with $Z=0.01$ and $Z=0.02$ in both cases $X=0.0$ (z1 and z2 in Table 3$)$ where $X, Y, Z$ are mass fractions of hydrogen, helium and metals. Mixtures with enhanced carbon and/or nitrogen (fv, n1, n2, c1 and c2 in Table 3) were also considered.

The models are described in Table 4 (see also Fig. 3 ).

One model (11) was computed for direct comparison with that of Fadeyev \& Lynas-Gray (1996). This model has the same stellar parameters as the model they consider to be in best agreement with observations at the time, i.e. $\log L / L_{\odot}=3.03, M / M_{\odot}=0.72, \log T_{\text {eff }}=4.37$ and mixture B13.002 (our mixture fv in Table 3). Our model has velocity and luminosity amplitudes similar to those obtained by them, showing smooth maxima in velocity and luminosity and a characteristic spike after the maximum in the luminosity curve. Although this model was already in good agreement with observations, new models were calculated specifically in an attempt to match the composition of $\mathrm{V} 652 \mathrm{Her}$ and either the lower mass or the radius suggested by recent observations (models 12-20).

Another five models were computed to match the composition of BX Cir and either the mass or the radius (models 21-25). Certain models were computed simply to explore further certain areas of the parameters space.
Table 3. Chemical composition adopted: relative abundances by number.

\begin{tabular}{cccccccc}
\hline & $\mathrm{fv}$ & $\mathrm{n} 1$ & $\mathrm{n} 2$ & $\mathrm{c} 1$ & $\mathrm{c} 2$ & $\mathrm{z} 1$ & $\mathrm{z} 2$ \\
$n_{\mathrm{H}}$ & 0.0060 & 0.0115 & 0.0076 & 0.0000 & 0.0000 & 0.0000 & 0.0000 \\
$n_{\mathrm{He}}$ & 0.9900 & 0.9862 & 0.9883 & 0.9961 & 0.9934 & 0.9976 & 0.9952 \\
$n_{\mathrm{N}}$ & 0.0020 & 0.0015 & 0.0027 & 0.0003 & 0.0005 & 0.0002 & 0.0003 \\
$n_{\mathrm{C}}$ & 0.0005 & $7 \times 10^{-5}$ & 0.0001 & 0.0029 & 0.0048 & 0.0006 & 0.0012 \\
$n_{\mathrm{Fe}}{ }^{(1)}$ & 4.72 & 2.37 & 4.17 & 2.49 & 4.18 & 5.19 & 10.45 \\
$n_{\mathrm{Z}}$ & 0.0040 & 0.0023 & 0.0041 & 0.0039 & 0.0066 & 0.0024 & 0.0048 \\
\hline
\end{tabular}

(1) $/ 10^{-5}$.

Table 4 also gives the corresponding value for the factor $Q$, the surface gravity, the pulsation period, $\Pi$, and the amplitudes of the radial velocity, $\Delta U_{\mathrm{ph}}$, and $\log L / L_{\odot}$, $\Delta L_{\mathrm{ph}}$, at the stellar photosphere. If the model is stable against pulsations $(12,13,21,22)$, as happens for those mixtures with very low iron abundances, the latter values are omitted. If the model was not computed until it reached a limiting amplitude, its amplitudes are preceded by a $<$ sign indicating an upper limit.

\subsection{Variations of velocity and luminosity curves}

The object of this investigation was to study whether the amplitudes and the shape of the stellar radial velocity and luminosity variations could help to determine the stellar properties.

Because the period is the parameter most accurately determined by observation, fixing it and assuming the factor $Q$ approximately constant allows us to fix the stellar luminosity and to compare models in a small range of temperatures and masses around the measured values.

The changes in the light and velocity curves for 0.42 and $0.90 M_{\odot}$, as functions of temperature are shown in Fig. 4.

For $0.42 M_{\odot}$, we have found that for the higher effective temperature, a characteristic bump appears after the luminosity maxima, which causes a flattening effect over the light curve at $\log T_{\text {eff }}=4.38$. This shape is similar to the one observed for BX Cir. Since this feature is almost negligible for higher masses (see luminosity curve for $0.52 M_{\odot}$ in Fig. 5) the hypothesis of BX Cir having mass less than (because luminosity amplitudes increase with the mass) or equal to $0.40 M_{\odot}$ and effective temperatures around $\log T_{\text {eff }}=4.38$ seems suitable. However, this feature disappears when varying the chemical composition, as is shown in Fig. 6 models 25 and 4 .

For $0.90 M_{\odot}$, both velocity and luminosity amplitudes increase when temperature decreases. In the velocity curves, maxima and minima are rounder than for lower masses, whereas luminosity curves are, in general, sharper. These effects can be observed in Fig. 5 for different stellar masses and approximately constant effective temperature, around $\log T_{\text {eff }} \sim 4.37$. 
Table 4 .

\begin{tabular}{|c|c|c|c|c|c|c|c|c|c|c|}
\hline Model & $\log L / L_{\odot}$ & $M / M_{\odot}$ & $\log T_{\text {eff }}$ & $R / R_{\odot}$ & $Q$ & $\log g$ & $\Pi / d$ & $\Delta U_{\mathrm{ph}} / \mathrm{km} \mathrm{s}^{-1}$ & $\Delta L_{\mathrm{ph}}$ & Compos. ${ }^{(1)}$ \\
\hline 1 & 2.879 & 0.42 & 4.380 & 1.592 & 0.0346 & 3.66 & 0.1073 & 127.17 & 0.16 & $\mathrm{z} 1$ \\
\hline 2 & 2.826 & 0.42 & 4.367 & 1.592 & 0.0345 & 3.66 & 0.1068 & 129.20 & 0.17 & z1 \\
\hline 3 & 2.719 & 0.42 & 4.340 & 1.591 & 0.0344 & 3.66 & 0.1065 & 98.02 & 0.17 & z1 \\
\hline 4 & 2.886 & 0.52 & 4.363 & 1.734 & 0.0336 & 3.68 & 0.1065 & 123.80 & 0.20 & z1 \\
\hline 5 & 3.142 & 0.70 & 4.400 & 1.965 & 0.0331 & 3.70 & 0.1090 & 118.33 & 0.20 & $\mathrm{z} 1$ \\
\hline 6 & 3.102 & 0.70 & 4.390 & 1.965 & 0.0331 & 3.70 & 0.1089 & 120.81 & 0.22 & z1 \\
\hline 7 & 3.210 & 0.90 & 4.400 & 2.125 & 0.0328 & 3.74 & 0.1072 & 107.73 & 0.24 & z1 \\
\hline 8 & 3.121 & 0.90 & 4.380 & 2.104 & 0.0328 & 3.75 & 0.1055 & 126.31 & 0.31 & z1 \\
\hline 9 & 3.089 & 0.90 & 4.370 & 2.123 & 0.0328 & 3.74 & 0.1070 & 130.85 & 0.32 & z1 \\
\hline 10 & 3.152 & 1.16 & 4.367 & 2.310 & 0.0327 & 3.78 & 0.1066 & 185.14 & 0.39 & z1 \\
\hline 11 & 3.026 & 0.72 & 4.371 & 1.965 & 0.0331 & 3.71 & 0.1074 & 104.75 & 0.22 & $\mathrm{fv}$ \\
\hline 12 & 2.886 & 0.52 & 4.363 & 1.734 & - & 3.68 & - & - & - & n1 \\
\hline 13 & 3.089 & 0.97 & 4.363 & 2.190 & - & 3.74 & - & - & - & $\mathrm{n} 1$ \\
\hline 14 & 2.890 & 0.62 & 4.350 & 1.851 & 0.0334 & 3.70 & 0.1068 & $<82.20$ & 0.17 & $\mathrm{n} 2$ \\
\hline 15 & 2.940 & 0.62 & 4.360 & 1.873 & 0.0333 & 3.69 & 0.1084 & 80.50 & 0.16 & $\mathrm{n} 2$ \\
\hline 16 & 2.920 & 0.60 & 4.360 & 1.830 & 0.0335 & 3.69 & 0.1070 & $<79.50$ & $<0.16$ & $\mathrm{n} 2$ \\
\hline 17 & 2.869 & 0.64 & 4.340 & 1.892 & 0.0333 & 3.69 & 0.1084 & $<69.28$ & $<0.15$ & $\mathrm{n} 2$ \\
\hline 18 & 2.950 & 0.64 & 4.360 & 1.894 & 0.0334 & 3.69 & 0.1088 & $<82.70$ & $<0.17$ & $\mathrm{n} 2$ \\
\hline 19 & 3.000 & 0.67 & 4.370 & 1.916 & 0.0333 & 3.70 & 0.1080 & $<83.67$ & $<0.17$ & $\mathrm{n} 2$ \\
\hline 20 & 3.010 & 0.70 & 4.370 & 1.938 & 0.0332 & 3.71 & 0.1071 & 84.69 & 0.17 & $\mathrm{n} 2$ \\
\hline 21 & 2.826 & 0.42 & 4.367 & 1.588 & - & 3.66 & - & - & - & $\mathrm{c} 1$ \\
\hline 22 & 3.152 & 1.16 & 4.367 & 2.310 & - & 3.77 & - & - & - & $\mathrm{c} 1$ \\
\hline 23 & 2.838 & 0.38 & 4.380 & 1.519 & 0.0351 & 3.65 & 0.1065 & 55.96 & 0.08 & $\mathrm{c} 2$ \\
\hline 24 & 2.740 & 0.40 & 4.350 & 1.558 & 0.0347 & 3.65 & 0.1065 & $<36.75$ & $<0.07$ & $\mathrm{c} 2$ \\
\hline 25 & 2.820 & 0.50 & 4.350 & 1.708 & 0.0338 & 3.67 & 0.1068 & 53.38 & 0.10 & $\mathrm{c} 2$ \\
\hline 26 & 3.136 & 0.90 & 4.380 & 2.140 & 0.0320 & 3.73 & 0.1056 & 170.68 & 0.27 & $\mathrm{z} 2$ \\
\hline
\end{tabular}

${ }^{(1)}$ See Table 3 .

Our experiment to investigate the effect of increased carbon and/or nitrogen content within the metal component was not entirely successful since in the cases with mixtures $\mathrm{n} 1$ and $\mathrm{c} 1$, closest to the measured ones, models were stable.

The reason is that the increase in carbon (or nitrogen) means a reduction in the iron-group elements (for constant total metallicity $n_{\mathrm{Z}}$ ). Therefore in these mixtures $\mathrm{n} 1$ and $\mathrm{c} 1, n_{\mathrm{Fe}}$ is approximately half the solar value. When $n_{\mathrm{Z}}$ is increased to 0.0041 (for the nitrogen-rich mixture, n2) and to 0.0066 (for the carbon-rich mixture, c2), the iron abundance increase to $n_{\mathrm{Fe}} \sim 4.17 \times 10^{-5}$ and the irongroup bump mechanism becomes effective. In these cases, models were unstable and velocity and luminosity curves were consistent with the observed ones.

The behaviour of velocity and luminosity with increasing iron abundance is illustrated in Fig. 6. The velocity curves show a faster growth and sharper maxima and minima when the iron abundance is higher (models 4, 6 and 26). These models also show a characteristic spike after the maximum in the luminosity curve.
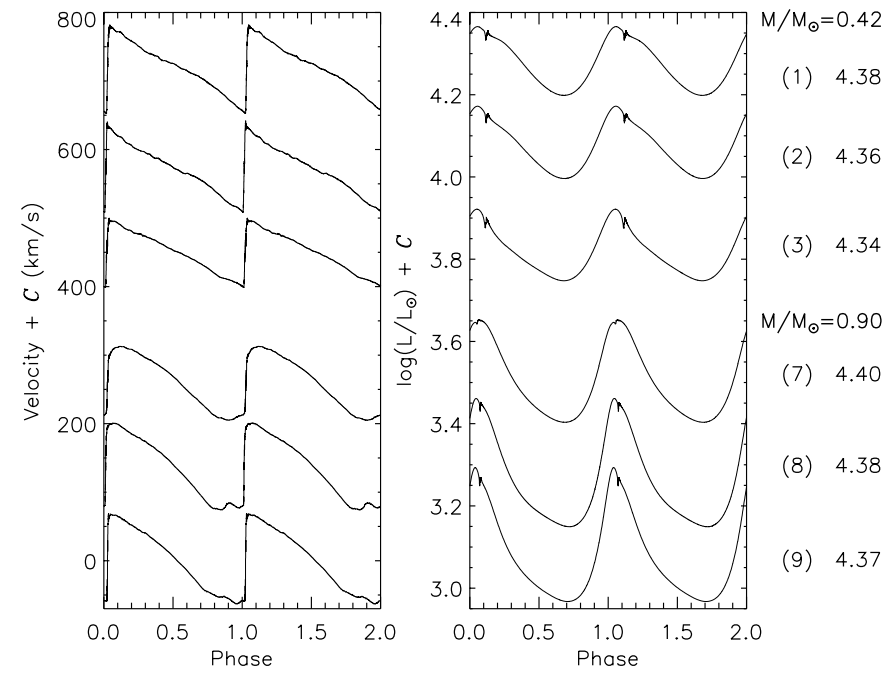

Fig. 4. Variations of the velocity and luminosity curves with effective temperature, for models with $M=0.42 M_{\odot}(1,2$ and 3$)$ and $M=0.90 M_{\odot}(7,8$ and 9$)$. Model number in Table 4 is indicated in brackets. In Figs. $4-7$ the vertical axes have been shifted by an arbitrary amount. 

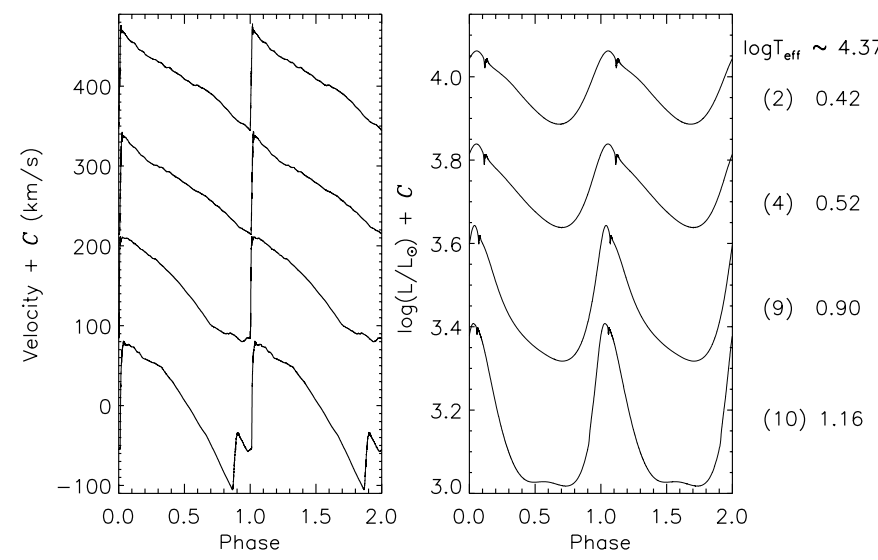

Fig. 5. Variations of the velocity and luminosity curves with stellar mass for effective temperature approximately constant $\left(\log T_{\text {eff }} \sim 4.37\right)$.

\section{Comparison with V652 Her and BX Cir}

The observed radial velocity and luminosity curves for V652 Her and BX Cir are shown in Fig. 7. The measured velocity and luminosity $\left(\Delta \log L / L_{\odot}\right)$ amplitudes are respectively $94 \mathrm{~km} \mathrm{~s}^{-1}$ and 0.240 for V652 Her and $56 \mathrm{~km} \mathrm{~s}^{-1}$ and 0.096 for BX Cir.

The observed radial velocities have been transformed into the stellar rest frame by multiplying the projection factor for helium-rich early-type stars, $p=-1.402$ (Montañés Rodríguez \& Jeffery 2001). Observational data have been taken from Jeffery et al. (2001: V652 Her) and Woolf \& Jeffery (2000: BX Cir).

Figure 7 also shows two models for each case, which fit quite well to the observed velocity and light curves, having not only a closer chemical composition to that observed but also similar parameters. For V652 Her these models have $0.62 M_{\odot}$ and $22900 \mathrm{~K}$ and $0.70 M_{\odot}$ and $23400 \mathrm{~K}$. The second (model 20) shows the best fit and has a radius closer to that given by observations, although a bigger mass.

For BX Cir, model 25 with $0.50 M_{\odot}$ and $22400 \mathrm{~K}$ presents a good fit reproducing not only the measured velocity and luminosity amplitudes but also the observed shapes, except for the flattening observed in the light curve which has not been reproduced in any case for this composition. Model 23 with a smaller mass, $0.38 M_{\odot}$, and higher temperature, $24000 \mathrm{~K}$, is also quite close to the observations for BX Cir. The velocity and luminosity curves of these two models are in remarkably good agreement with the observed ones. We emphasize that, after the pulsation period, they are the most directly and reliably measured properties of BX Cir.

\section{Conclusion}

In this paper we provide new non-linear radial pulsation models computed within the iron-group instability strip where low-mass extreme-helium stars are located. We attempt to reproduce the observed light and velocity curves
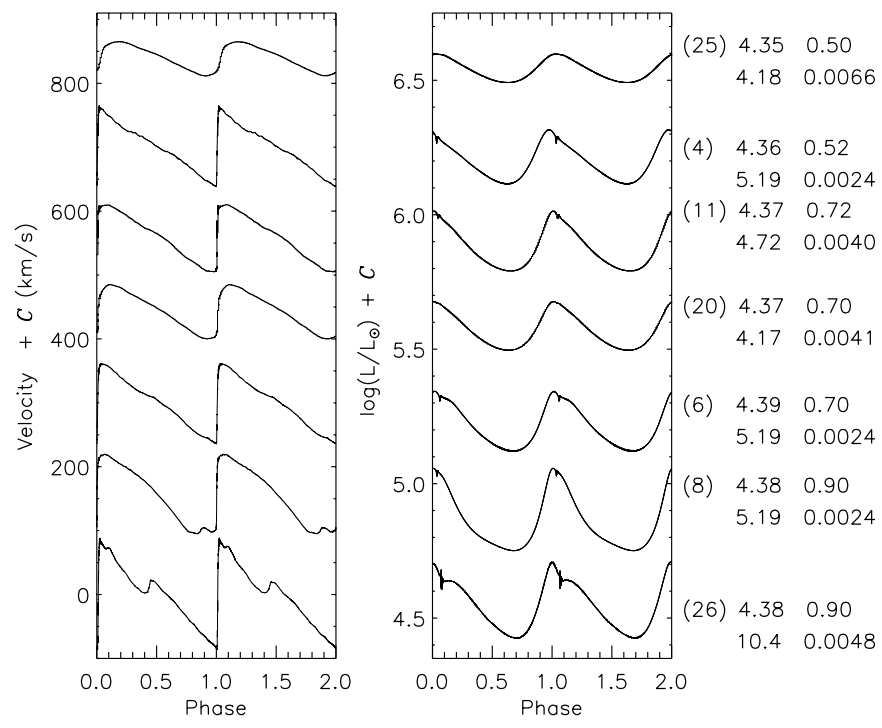

Fig. 6. Velocity and luminosity curves for models indicated into brackets. Next to the model number: $\log T_{\text {eff }}$, stellar mass $\left(M_{\odot}\right)$, iron abundance $\left(n_{\mathrm{Fe}}\right)$ and total abundance of metals $\left(n_{\mathrm{Z}}\right)$ appear respectively.

of V652 Her and BX Cir and, hence, study their possible masses, luminosities and radii.

All the models have temperatures and masses close to those measured for V652 Her and BX Cir. Their luminosities are chosen to ensure the pulsation period matches that observed. The chemical composition was initially chosen to be similar to the observed surface composition, but this produces stable models. Metallicity was gradually increased to find unstable (overstable) models, which provide a lower limit to the iron abundance of around $n_{\mathrm{Fe}} \sim 4.17 \times 10^{-5}$. This indicates that the recently published iron abundance BX Cir may be too small, although a more detailed study of the dependence of the instability boundaries on the iron abundance using a linear analysis is recommended. It is evident that the pulsation properties (amplitude, light curve shape) are very sensitive to the chemical composition.

We have found that several models can reproduce with good accuracy the observed velocity and luminosity curves for these two stars.

The parameters of the best fit model for V652 Her are close to those previously provided by Fadeyev \& Lynas-Gray (1996) but with lower helium, carbon and iron abundances (model 20) more similar to those reported in a recent spectroscopic analysis (Jeffery et al. 2001). An attempt to reproduce the velocity and light curves with a lower mass $\left(0.59-0.67 M_{\odot}\right)$, closer to that provided by recent observations, within the range of temperatures $21900-23400 \mathrm{~K}$ was not successful. For $T_{\text {eff }} \sim 23400 \mathrm{~K}$, a mass of at least $0.70 M_{\odot}$ is necessary to reproduce the velocity and luminosity curves. This value would be slightly higher for lower temperatures. The white dwarf merger model (Saio \& Jeffery 2000) introduced to account for the origin of V652 Her requires a final mass between 

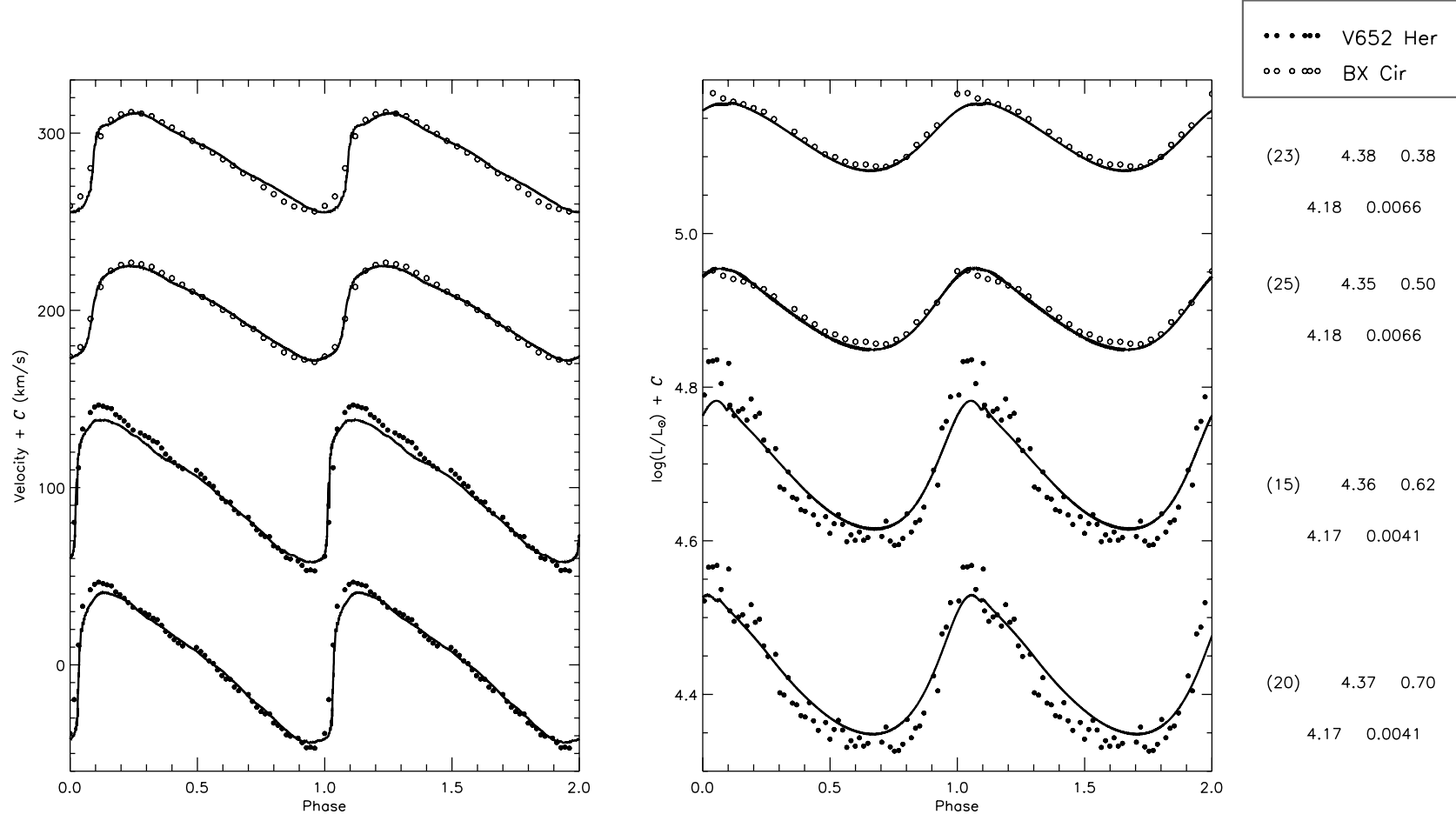

Fig. 7. Velocity and luminosity curves for four selected models with mixtures $\mathrm{n} 2$ and $\mathrm{c} 2$. Next to the model number: $\log T_{\text {eff }}$, stellar mass $\left(M_{\odot}\right)$, iron abundance $\left(n_{\mathrm{Fe}}\right)$ and total abundance of metals $\left(n_{\mathrm{Z}}\right)$ appear respectively.

0.6 and $0.7 M_{\odot}$, which is in satisfactory agreement with the results of our pulsation analysis.

As suggested by Jeffery et al. (2001), it remains a problem to reconcile fully the optical line spectrum, the overall flux distribution and, as here, the pulsation properties of V652 Her.

The pulsation period and velocity and luminosity amplitudes of BX Cir could be satisfactorily reproduced by a model (25) with a mass and temperature within the observational errors. A minimum mass of $0.38 M_{\odot}$ for $23900 \mathrm{~K}$ and a maximum mass of $0.50 M_{\odot}$ for $22300 \mathrm{~K}$ were found. Between these limits, an increase in stellar mass requires a corresponding decrease in effective temperature in order to reproduce the observed velocity and luminosity amplitudes.

It is not possible to match the observed luminosity for BX Cir, which seems too large by a factor of $\sim 2$. The reason is far from clear because both temperature and radius are well defined by observation (Woolf \& Jeffery 2000). Pulsation theory imposes a very tight relation between pulsation period and mean density - or mass-to-radius ratio. Small departures from this ratio do not significantly alter the shapes of light and velocity curves. Thus we believe that fixing the period is the most appropriate way to approach the problem. There are several other possibilities. One is that the radius of Woolf \& Jeffery (2000) is too big, but their data do not seem to allow this. Another is that systematic errors in the model atmospheres overestimate the effective temperature and a smaller radius results. A third is that similar errors led to an underestimate of the surface gravity of Drilling et al. (1996) so that a higher mass would result. Finally, systematic errors in the pulsation models could yield incorrect amplitudes. Such errors would have to be selective in order to modify the BX Cir result more than the V652 Her result, and could occur if the composition adopted for either star were incorrect. Further observational work might yield evidence for a solution with a higher mass. This would be easier to reconcile with white dwarf merger models (Saio \& Jeffery 2000), which require masses in excess of $\sim 0.5 M_{\odot}$. However the conclusion of this paper is that the current pulsation models favour a low-mass solution for BX Cir. Otherwise the theoretical velocity amplitude is too large compared with that observed.

Acknowledgements. This research is supported by a grant to the Armagh Observatory from the Northern Ireland Department of Culture, Arts and Leisure and by the British Council through Collaborative Research Grant TOK $/ 880 / 41 / 4$. We are grateful to Prof. H. Saio (Tohoku University, Sendai) for valuable advice, to Prof. Y. A. Fadeyev (Institute of Astronomy of the RAS, Moscow) for his comments, to Dr. A. Bridger (UK Astronomy Technology Centre, Edinburgh) for his pulsation code and to the referee Dr. T. Metcalfe for his review of the paper.

\section{References}

Anders, E., \& Grevesse, N. 1989, Geochim. Cosmochim. Acta, 53,197

Alexander, D. R., \& Ferguson, J. W. 1994, ApJ, 437, 879A

Bridger, A. 1984, Cepheids: Theory \& Observations, IAU Coll., 82,246

Christy, F. 1966, ARA\&A, 4, 353 
Drilling, J. S., Jeffery, C. S., \& Heber, U. 1998, A\&A, 329, 1019 Drilling, J. S. 1980, ApJ, 242, L43

Fadeyev, Yu. A., \& Lynas-Gray, A. E. 1996, MNRAS, 280, 427 Grevesse, N., \& Noel, A. 1993, Origin and Evolution of the Elements, ed. N. Pratzo, E. Vangioni-Flam, \& M. Casse (Cambridge: Cambridge Univ. Press), 15 (GN93)

Hill, P. W., Kilkenny, D., Schönberner, D., \& Walker, H. J. 1981, MNRAS, 197, 81

Holweger, H., Heise, C., \& Kock, M. 1990, AA, 232, 510

Iglesias, C. A., \& Rogers, F. J. 1996, ApJ, 464, 943

Jeffery, C. S., \& Heber, U. 1992, A\&A, 260, 133

Jeffery, C. S. 1996, ASP Conf. Ser., 96, 152

Jeffery, C. S., \& Saio, H. 1999, MNRAS, 308, 221

Jeffery, C. S., Hill, P. W., \& Heber, U. 1999, A\&A, 346, 491

Jeffery, C. S., Woolf, V. M., \& Pollacco, D. 2001, A\&A, 376, 497
Kilkenny, D., \& Koen, C. 1995, MNRAS, 275, 327

Kilkenny, D., Koen, C., Jeffery, C. S., Hill, N. C., \& O'Donoghue, D. 1999, MNRAS, 310, 1119

Landolt, A. U. 1975, ApJ, 196, 789

Lynas-Gray, A. E., Schönberner, D., Hill, P. W., \& Heber, U. 1984, MNRAS, 209, 387

Montañés Rodríguez, P., \& Jeffery, C. S. 2001, A\&A, 375, 411

Saio, H. 1985, ApJ, 295, 38S

Saio, H. 1990, ASP Conf. Ser., 557

Saio, H. 1993, MNRAS, 260, 465

Saio, H. 1995, MNRAS, 277, 1393

Saio, H., \& Jeffery, C. S. 2000, MNRAS, 313, 671

Stellingwerf, R. F. 1975, ApJ, 195, 441

Stürenburg, S., \& Holweger, H. 1990, A\&A, 237, 125

Woolf, V. M., \& Jeffery, C. S. 2000, A\&A, 358, 1001 